\title{
Efficacy of Rosuvastatin and Atorvastatin in Vietnamese Patients with Acute Coronary Syndrome: A randomized trial
}

\author{
An Viet Tran ${ }^{1}$, Thanh Truong Nguyen ${ }^{2}$, Lien Nguyen Thao Tran ${ }^{1}$, Phuong Minh Nguyen ${ }^{1}$, Thang Nguyen ${ }^{3}$ \\ 1 Faculty of Medicine, Can Tho University of Medicine and Pharmacy, Can Tho, Vietnam \\ 2 Soc Trang General Hospital, Soc Trang Province, Vietnam \\ 3 Faculty of Pharmacy, Can Tho University of Medicine and Pharmacy, Can Tho, Vietnam
}

\begin{abstract}
High-intensity statins have been recommended to initiate in patients after acute coronary syndrome (ACS) to reduce the risk of death and recurrent cardiovascular events. There have been few studies comparing the effectiveness of high-intensity statins in Vietnam. Therefore, we conducted a randomized controlled trial to compare the effects of rosuvastatin versus atorvastatin in Vietnamese patients with ACS. A total of 96 ACS patients were randomized into 2 groups at a ratio of $1: 1$ to receive rosuvastatin $20 \mathrm{mg}$ /day or atorvastatin 40 $\mathrm{mg} /$ day in addition to the standard treatment of ACS. LDL-c and hs-CRP levels were measured before and after the intervention for analysis. No significant differences were found between the two groups at baseline. LDL-c levels were significantly reduced from baseline to 4-day in both groups ( $p<0.001$ in both groups), but the difference in LDL-c levels at 4-day between the groups was not statistically significant ( $p=0.251)$. hs-CRP did not increase significantly in the rosuvastatin group but significantly in the atorvastatin group $(p=0.209$ and $p<0.001$, respectively). hs-CRP at 4-day was not significantly different between the two groups $(p=0.250)$. The proportion of $\mathrm{LDL}<1.8 \mathrm{mmol} / \mathrm{L}$ after 4 days in the rosuvastatin group was significantly higher than that of the atorvastatin group ( $\mathrm{OR}=4.592 ; 95 \% \mathrm{CI} 1.365-15.449 ; \mathrm{p}=0.014)$. There was no significant difference in the LDL-c reduction $\geq 50 \%$ and hs-CRP $\leq 3 \mathrm{mg} / \mathrm{L}$ at 4 -day between two groups. In conclusion, rosuvastatin is more effective than atorvastatin in achieving LDL-c targets of $<1.8 \mathrm{mmol} / \mathrm{L}$ after 4 days in Vietnamese patients with ACS.
\end{abstract}

\section{Keywords:}

Acute coronary syndrome, Atorvastatin, High-sensitivity C-reactive protein (hs-CRP), Low-density lipoprotein cholesterol (LDL-c), Rosuvastatin.

\section{INTRODUCTION}

Ischemic heart disease, including acute coronary syndrome (ACS), is the leading cause of morbidity and mortality worldwide. It caused more than 9 million deaths globally in 2016, according to World Health Organization statistics ${ }^{1,2}$. The increase of low-density lipoprotein cholesterol (LDL-c) and high-sensitivity Creactive protein (hs-CRP) in patients with ACS has been shown to be associated with the increase of mortality and recurrent adverse cardiovascular events ${ }^{3-5}$. Therefore, reducing LDL-c and hs-CRP have become important targets for treatment in $\mathrm{ACS}^{6-8}$.

Statins have been recommended as the first choice to lower LDL-c levels ${ }^{6}$. Statins reduce the synthesis of cholesterol in the liver by competitively inhibiting 3-hydroxyl-3-methylglutaryl coenzyme A reductase activity. Statins are effective in reducing triglycerides, LDL-c and total cholesterol. Besides, they also slightly increase high-density lipoprotein cholesterol $^{9}$. In addition to their beneficial effects on blood lipids, statins have been shown to reduce hs-CRP levels ${ }^{10}$.

American and European Guidelines have recommended initiating high-intensity statins as soon as

\section{*Corresponding author:}

*An Viet Tran tvan@ctump.edu.vn 
possible and prolonged maintaining in patients after ACS to cut down the risk of death and secondary cardiovascular events, regardless of baseline LDL- $c^{6}$, $8,11-13$. The target for LDL-c is $<1.8 \mathrm{mmol} / \mathrm{L}(70 \mathrm{mg} / \mathrm{dL})$ or LDL-c reduction by at least $50 \%$ of the baseline value $^{12,13}$. High-intensity statins are the statins that effectively lower LDL-c by approximately $>50 \%$, including atorvastatin at doses of $40 \mathrm{mg} / \mathrm{day}$ or 80 $\mathrm{mg} / \mathrm{day}$; and rosuvastatin at doses of $20 \mathrm{mg} /$ day or 40 $\mathrm{mg} /$ day $^{14}$.

Different statins with different doses have different levels of LDL-c reduction. There are different levels of response among individuals even with the same dose of statin. The variation in response to statin treatment may be due to differences in genes that regulate cholesterol metabolism as well as statin absorption and metabolism in the liver ${ }^{13}$.

In recent years, several studies have been done to compare the effects of high-intensity statins in patients after ACS around the world. Rosuvastatin seems to be more effective than atorvastatin in reducing LDL-c and hs-CRP levels ${ }^{15,16}$. However, very few clinical trials have been conducted in Vietnam to discuss this issue. Therefore, we conducted this study with the purpose of comparing the effects of rosuvastatin versus atorvastatin in Vietnamese patients after acute coronary syndrome.

\section{MATERIALS AND METHODS}

\subsection{Study design and setting}

We conducted an open-label, parallel-group, single-center, randomized trial to compare the effects of treatment with rosuvastatin versus atorvastatin in Vietnamese patients after acute coronary syndrome. The study was performed at Can Tho University of Medicine and Pharmacy Hospital in Vietnam from June 2017 to April 2019.

\subsection{Study population}

The study population consisted of patients over 18 years of age in both genders admitted to the hospital with the diagnoses of acute coronary syndrome and with baseline LDL-c levels of $1.8 \mathrm{mmol} / \mathrm{L}$ and above. Acute coronary syndrome included ST elevation myocardial infarction (STEMI), non-ST elevation myocardial infarction (NSTEMI), or unstable angina (UA) according to the European Society of Cardiology criteria ${ }^{6,8}$.

We excluded patients who were already using statins and/or other hypolipidemic drugs, and/or any other drug which strongly inhibits CYP3A4 (antiretroviral drugs, ketoconazole, itraconazole, clarithromycin) or CYP2C9 (fluconazole), patients with any history of hypersensitivity or allergy to statins or any contraindication to the use of statins, patients with severe cardiac dysfunction (ejection fraction $<30 \%$ ), and any other comorbidity including severe anemia, malignancy, chronic liver disease, chronic renal failure, pregnancy or lactation. Patients who had a history of operation or injury within 2 months were also excluded from the study.

\subsection{Sample size}

The sample size of this study was calculated based on information from the study on the efficacy and tolerability of rosuvastatin and atorvastatin in patients with primary hypercholesterolemia ${ }^{17}$. In the study, the proportion of LDL-c $<1.8 \mathrm{mmol} / \mathrm{L}$ after treatment with high doses of rosuvastatin and atorvastatin in the highrisk group was estimated at $37 \%$ and $12 \%$, respectively. We assumed that the probability of a type 1 error (alpha) was 0.05 (95\% confidence level) and that type 2 error (beta) was 0.20 ( $80 \%$ power). Therefore, the minimum sample size needed for each group was 36 participants. Nevertheless, to prevent loss of follow-up, the sample size was increased by $20 \%$. In fact, we recruited 48 patients per group.

\subsection{Randomization and intervention}

Patients who met the criteria of the study and signed the informed consent were randomized at a 1:1 ratio into either group A or group B based on their inpatient code. Each patient has an inpatient code provided by the nurses from the clinic on admission. Group A included the patients whose inpatient codes were even numbers. Group B included the patients whose inpatient codes were odd numbers. Group A received $20 \mathrm{mg}$ rosuvastatin daily and group $B$ received $40 \mathrm{mg}$ atorvastatin daily along with standard treatment including aspirin, clopidogrel, beta-blockers, nitrates, and an angiotensin-converting enzyme inhibitor.

\subsection{Data collection}

Firstly, we collected data from medical records and via face to face interviews included general characteristics (age, gender, body mass index), coronary artery disease risk factors (hypertension, diabetes, dyslipidemia, lack of physical activity, overweight or obesity, smoking, early coronary artery disease family history), medical history and comorbidities (current medications, drug allergies, severe anemia, severe heart failure, malignancy, chronic renal failure, chronic liver disease, pregnancy or lactation, operation or injury within 2 months) and diagnosis. Serum low-density lipoprotein cholesterol (LDL-c) and high-sensitivity Creactive protein (hs-CRP) were recorded for all patients 
at baseline (before starting therapy). Next, eligible patients and their relatives were consulted to agree on participation in the study. They were clearly explained the objectives and process of the research. The patients who voluntarily took part in the study signed into informed consent. Then, these patients were randomized into intervention groups. Finally, serum LDL-c and hsCRP were recorded again after four-day treatment.

Serum LDL-c levels were measured by the chemistry autoanalyzer (ARCHITECT ci4100, Abbott Diagnostics, USA) via enzymatic colorimetric methods. Serum hs-CPR levels were determined with the enzyme-linked immunosorbent assay technique by the chemistry autoanalyzer (ARCHITECT ci4100, Abbott Diagnostics, USA).

\subsection{Study outcomes}

Primary outcome measures included levels of hs-CRP and LDL-c after 4 days of treatment. Secondary outcomes included (1) the proportion of patients achieving the LDL-c goal of $<1.8 \mathrm{mmol} / \mathrm{L}$ after 4 days; (2) the proportion of patients achieving an LDL-c reduction of at least 50\%; (3) proportion of patients with hs-CRP $\leq 3 \mathrm{mg} / \mathrm{L}$ after 4 days.

\subsection{Statistical analysis}

Statistical analyses were performed using the Statistical Package for the Social Sciences, version 20th (SPSS 20). The distribution of continuous variables was tested using the Kolmogorov-Smirnov test. Continuous variables were presented as mean \pm standard deviation if normally distributed and as median (interquartile range) if non-normally distributed. Categorical variables were recorded as frequency (percentage). Continuous variables with normal distribution were compared within groups by using independent sample t-test and between two groups by using paired sample t-test. Continuous variables without normal distribution were compared within groups by using the Mann-Whitney's U test and between two groups by using Wilcoxon signed-rank test. Categorical variables were compared using Chi-square test. Univariable and multivariable logistic regression models were used to estimate the odds ratio (OR) with $95 \%$ confidence interval $(\mathrm{CI})$ of the outcomes. The variables entered on step 1 of the multivariate analysis included intervention status, age and gender. All tests were two-sided. A $p$-value $<0.05$ was considered to be statistically significant for all tests.

\subsection{Ethical approval}

The study was approved by the biomedical research ethics committee and the management board of the study hospital in May 2017 with the number 34.2017/HDDD-DHYDCT.

\section{RESULTS}

A total of 106 patients presenting with ACS were assessed for eligibility. Of these, 96 (90.6\%) patients were included; $10(9.4 \%)$ patients were excluded due to exclusion criteria. Of 96 patients included, 48 were randomized to the group received rosuvastatin $20 \mathrm{mg}$ daily and 48 to the group received atorvastatin 40mg daily. The follow-up took 4 days. All patients were measured serum LDL-c and hs-CRP levels before and after intervention for analysis (Figure 1).

The mean age (SD) was 63.0 (12.0) years and patients aged $\geq 60$ years were more common $(61.5 \%)$. $67.7 \%$ were males and median (IQR) Body Mass Index was $23.7(21.8 ; 24.6) \mathrm{kg} / \mathrm{m}^{2}$. Risk factors for coronary artery disease that predominated in our patients included hypertension, dyslipidemia, physical inactivity, overweight or obesity and smoking. The majority of patients had a diagnosis of non-ST elevation acute coronary syndrome (53.1\%). Mean LDL-c level was 3.38 $\mathrm{mmol} / \mathrm{L}$ with standard deviation of $0.92 \mathrm{mmol} / \mathrm{L}$. Median hs-CRP level of these patients was $4.80 \mathrm{mg} / \mathrm{L}$ (interquartile range 1.55 to $10.25 \mathrm{mg} / \mathrm{L}$ ). The baseline characteristics of the groups are presented in Table 1, and there were no significant differences between the two groups.

There was a significant decrease in mean serum LDL-c levels from baseline to 4 days in both groups: group A ( $3.32 \pm 0.81$ vs. $2.24 \pm 0.81, p<0.001)$ and group B $(3.45 \pm 1.02$ vs. $2.41 \pm 0.67, p<0.001)$. Unlike LDL-c levels, median serum hs-CRP levels significantly increased from baseline to 4 days in group B [4.40 (1.30; $8.38)$ vs. $11.58(5.12 ; 24.86), p<0.001]$. There was also an increase in median serum hs-CRP levels from baseline to 4 days in group A but this increase was not statistically significant $[5.00(2.13 ; 15.55)$ vs. 8.65 (3.16; 20.85), $p=0.209$ ]. Mean serum LDL-c levels and median serum hs-CRP levels were not significantly different between the 2 groups at 4 days $(p=0.251$ and $p=0.250$, respectively) (Table 2).

The proportion of patients with serum LDL-c levels $<1.8 \mathrm{mmol} / \mathrm{L}$ at 4 days was significantly higher in group A than group B $(\mathrm{OR}=4.592,95 \% \mathrm{CI}: 1.365$ 15.449, $p=0.014)$. The proportions of patients with LDL-c reduction at least 50\% compared with baseline levels and hs-CRP levels $\leq 3 \mathrm{mg} / \mathrm{L}$ at 4 days were higher in group A than group B (OR=3.007, 95\% CI 0.942 - 9.599 and $\mathrm{OR}=1.818,95 \%$ CI 0.596 - 5.542, respectively). Nevertheless, these were not significantly different between the 2 groups $(p=0.063$ and $p=0.293$, respectively) (Table 3). 

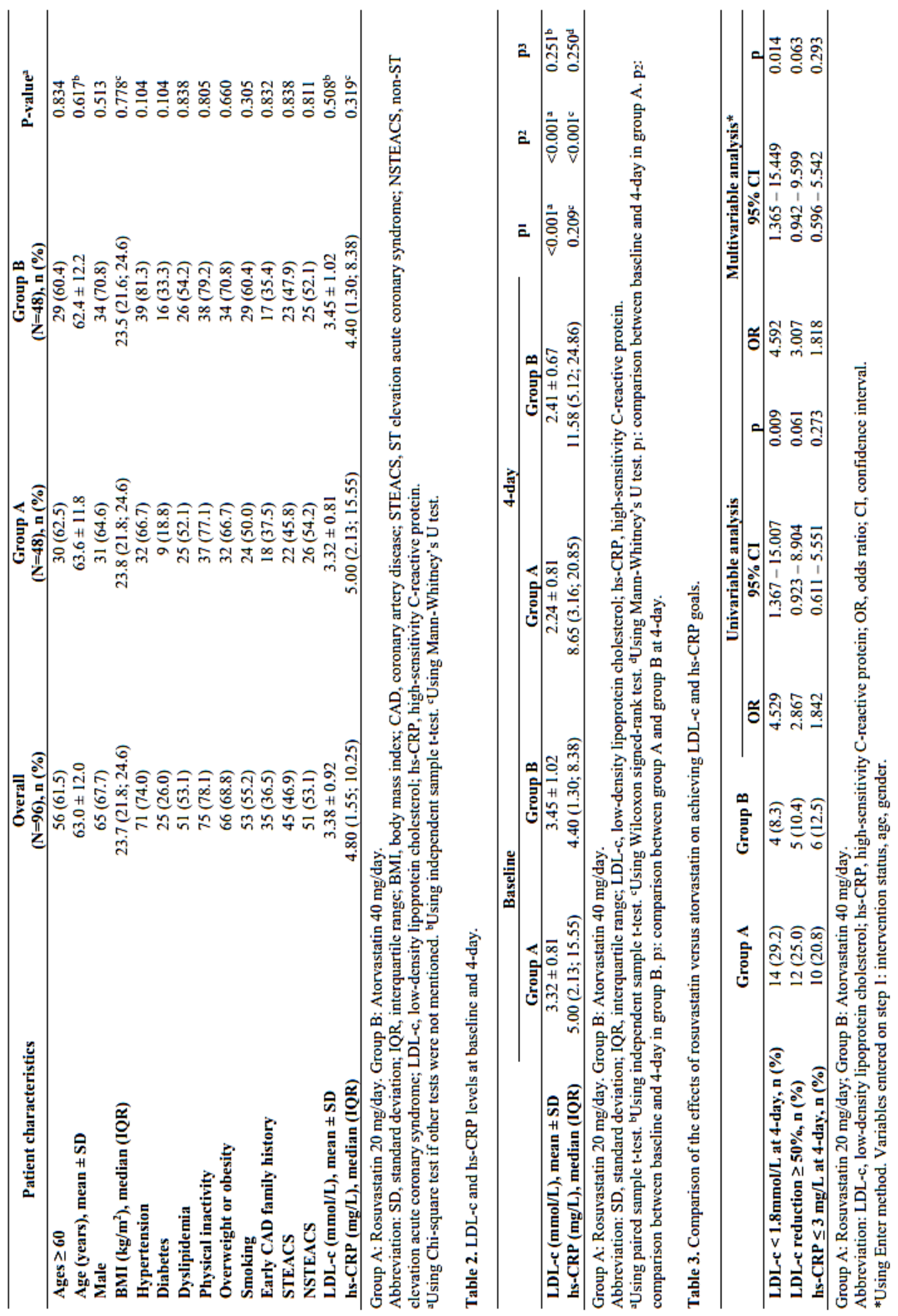


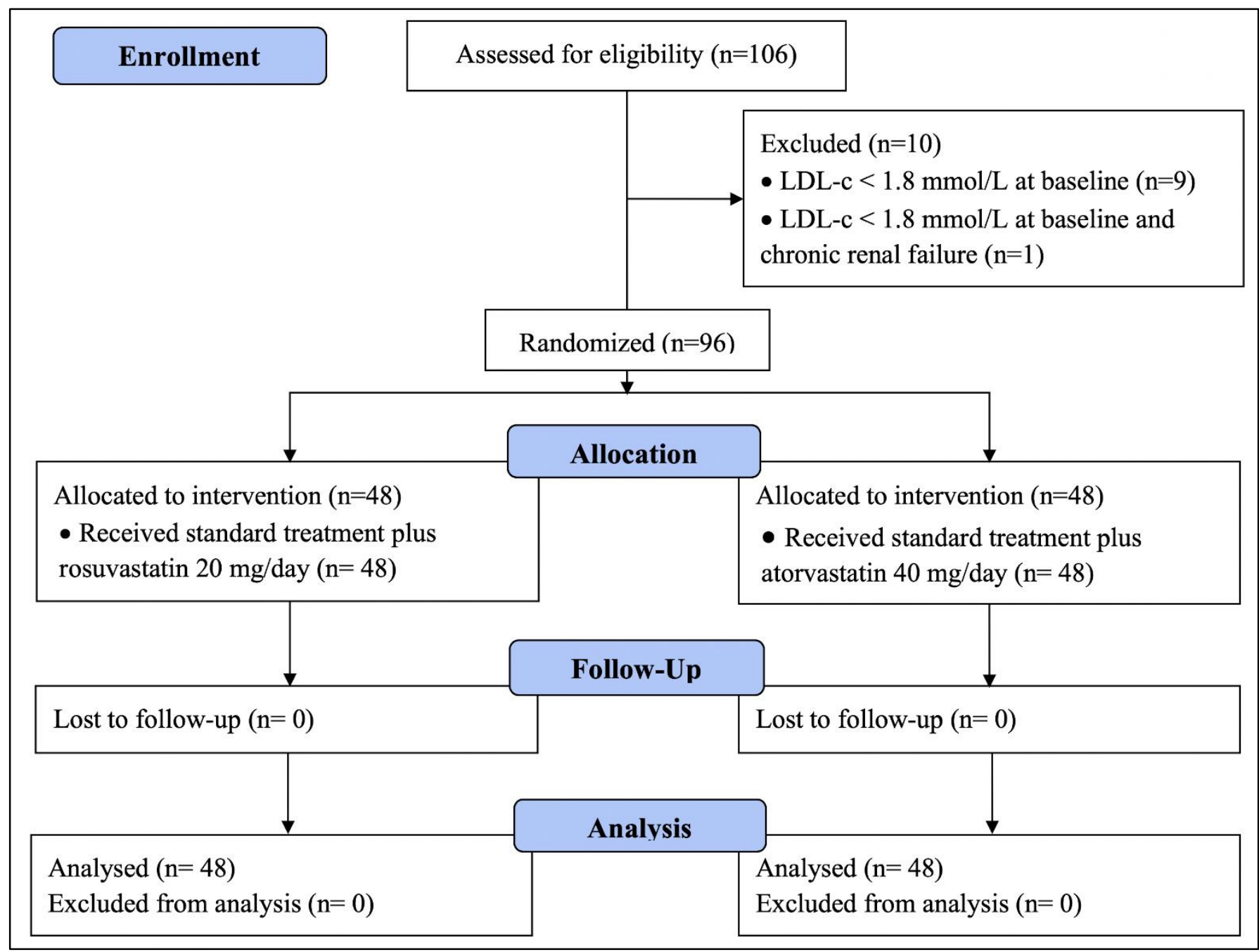

Figure 1. Flow diagram of the study population Abbreviation: LDL-c, low-density lipoprotein cholesterol.

\section{DISCUSSION}

This study was a clinical trial with 2 intervention groups randomly assigned at a ratio of $1: 1$ to receive rosuvastatin $20 \mathrm{mg} /$ day or atorvastatin 40 $\mathrm{mg} / \mathrm{day}$. There was no statistically significant difference between the two study groups in the demographic characteristics, coronary artery disease risk factors, type of ACS, LDL-c and hs-CRP levels at baseline. The observation period of the study was only 4 days. All patients remained in the hospital for treatment, so no patients were lost to follow-up or dropped out of the study.

Several studies showed that LDL-c levels dropped within the first 24 to 48 hours, reached a maximum within 4 to 7 days after admission ${ }^{18,19}$. Other studies have reported that hs-CRP levels increase after admission and peak within 2-4 days ${ }^{20,21}$. Therefore, we used LDL-c and hs-CRP levels on day 4 to evaluate the early efficacy of high-intensity statins in reducing these biomarkers.

Our study has supported the role of statins in lowering serum LDL-c levels. After only 4 days of treatment, LDL-c levels were significantly reduced in both groups treated with rosuvastatin and atorvastatin. Previous studies with longer follow-up times have shown similar results. An open-label randomized clinical trial conducted on 100 patients with ACS showed that both rosuvastatin $20 \mathrm{mg}$ and atorvastatin $40 \mathrm{mg}$ were effective in lowering LDL-c levels after 4 weeks ${ }^{22}$. Another study with the same 4-week observation period but higher doses of statins also showed a significant reduction in LDL-c levels ${ }^{23}$. Our study has not demonstrated a significant difference in LDL-c levels at 4-day between the two intervention groups. A few previous studies have also reached a similar conclusion that atorvastatin and rosuvastatin reduced LDL-c levels equally ${ }^{22,24}$. The results from LUNAR study showed that compared to atorvastatin $80 \mathrm{mg}$, rosuvastatin $40 \mathrm{mg}$ was significantly more effective in reducing LDL-c, but rosuvastatin $20 \mathrm{mg}$ was equally effective ${ }^{16}$. It is deduced that despite being high-intensity statins, different doses of statins can have different therapeutic effects. In our study, the percentage of patients achieving the LDL-c goal of 
$<1.8 \mathrm{mmol} / \mathrm{L}$ after 4 days of the rosuvastatin group was significantly higher than the atorvastatin group. Our study may be one of the few studies that used the proportion of patients achieving the LDL-c goal of $<1.8$ $\mathrm{mmol} / \mathrm{L}$ to compare the effects of rosuvastatin versus atorvastatin.

The role of statins in reducing hs-CRP was not found in this study. After 4 days of treatment, hs-CRP levels increased in both groups. However, the increase in hs-CRP from baseline to 4-day treatment was not significant in the rosuvastatin group but significant in the atorvastatin group. This has shown that rosuvastatin may have been more effective than atorvastatin in limiting the increase in hs-CRP. Nevertheless, this difference was not really clear. This may be because our research period is too short. In other studies, the effect of decreasing hs-CRP of the statins was evident after 4 weeks, 6 weeks, and 12 weeks of treatment ${ }^{15,25}$.

There were differences in follow-up time in the studies. The follow-up time in our study was 4 days. Meanwhile, other studies had a longer follow-up period ${ }^{15}$, 16,24 . The duration of treatment may have affected the efficiency of statins. A previous study was performed to evaluate the efficacy and safety of rosuvastatin $40 \mathrm{mg}^{25}$. In the study, the hs-CRP level was not significantly reduced at 6 weeks from the baseline. However, a significant reduction in hs-CRP levels was observed after 12 weeks. Dosages of statins were also different among studies. Our study used a dose of rosuvastatin 20 $\mathrm{mg}$ daily and atorvastatin $40 \mathrm{mg}$ daily. In other studies, the dose of statins (rosuvastatin/atorvastatin) used was $40 / 80$ or $40 / 20 \mathrm{mg}$ daily ${ }^{15,23,24}$. Different doses of statins may have produced different results. A study was conducted to compare the effects of different doses of rosuvastatin $^{26}$. The study had three groups including the non-statin group, the rosuvastatin $10 \mathrm{mg} /$ day group, and the rosuvastatin $20 \mathrm{mg} /$ day group. The hs-CRP level of patients in 3 groups did not differ significantly after 1 week. Nevertheless, after 6 weeks and 12 weeks, the hs-CRP level in the rosuvastatin $20 \mathrm{mg}$ group was significantly lower than the other groups.

There were several limitations to our study. This study was only conducted on patients in a hospital and thus, the results may not represent all Vietnamese residents from other areas. The sample size of the study was small and the monitoring time was short, so the difference in efficacy of the statins could not be clearly seen. Further studies should be conducted with larger sample sizes, longer follow-up and multiple centers to demonstrate a significant superiority of rosuvastatin over atorvastatin in achieving LDL-c and hs-CRP treatment goals as well as preventions recurrent cardiovascular events in patients after ACS in Vietnam. The short follow-up may not be the best for assessing patients' outcomes. Because the hospital stay of the inpatient was not long enough to monitor the patient's long-term outcomes and there were some financial and timeframe limitations of the study, the comparison of the efficacy of rosuvastatin versus atorvastatin in this study was based only on early changes in LDL-c and hs-CRP levels. The evaluation of the long-term effect of statins on patient outcomes should be considered in future studies. The changes in LDL-c and hs-CRP levels may be affected by confounding factors including age, gender, type of ACS, interventions, patient adherence, etc. Further studies should consider confounding factors and perform subgroup analysis to assess their effects on study results. For clinical practice, rosuvastatin should be used more than atorvastatin in patients after ACS to achieve LDL-c goal of $<1.8 \mathrm{mmol} / \mathrm{L}$.

\section{CONCLUSIONS}

Rosuvastatin $20 \mathrm{mg}$ daily and atorvastatin 40 mg daily were both significantly effective in reducing LDL-c after 4 days in patients with ACS, but not with hs-CRP. Differences between the groups were not statistically significant. Rosuvastatin is more effective than atorvastatin in achieving LDC-c goals of 1.8 $\mathrm{mmol} / \mathrm{L}$ after 4 days, but not in reducing LDL-c levels by at least $50 \%$ and reaching hs-CRP levels of $\leq 3$ $\mathrm{mg} / \mathrm{dL}$. This study supported clinicians to choose rosuvastatin rather than atorvastatin to attain LDL-c levels of $<1.8 \mathrm{mmol} / \mathrm{L}$ in Vietnamese patients with ACS. Further studies with longer follow-up and larger sample sizes should be conducted to assess the longterm effects of the high-intensity statins on reducing LDL-c, hs-CRP levels as well as prevention recurrent cardiovascular events in Vietnamese patients post-ACS.

\section{ACKNOWLEDGEMENT}

We would like to express our sincere gratitude to Can Tho University of Medicine and Pharmacy Hospital for allowing us to conduct this study.

\section{Conflict of interest}

The authors declare that there are no conflicts of interest.

\section{Funding}

None to declare

\section{Ethical approval}

The study was approved by the biomedical research ethics committee and the management board of the study hospital in May 2017 with the number 34.2017/ HDDD-DHYDCT.

\section{Article info:}

Received August 2, 2020

Received in revised form October 20, 2020 
Accepted November 13, 2020

\section{REFERENCES}

1. Global Health Estimates 2016: Deaths by Cause, Age, Sex, by Country and by Region, 2000-2016. Geneva: World Health Organization; 2018; Available from: https://www.who.int/ healthinfo/global_burden_disease/estimates/en/.

2. Nowbar AN, Gitto M, Howard JP, Francis DP, Al-Lamee R. Mortality From Ischemic Heart Disease. Circ Cardiovasc Qual Outcomes. 2019;12(6):e005375.

3. Kawada-Watanabe E, Yamaguchi J, Sekiguchi H, Arashi H, Ogawa H, Hagiwara N. Targeting high-sensitivity C-reactive protein levels in acute coronary syndrome patients undergoing contemporary lipid-lowering therapy: a sub-analysis of the HIJPROPER trial. J Cardiol. 2020;75(5):500-6.

4. Pearson TA, Mensah GA, Alexander RW, Anderson JL, Cannon RO 3rd, Criqui M, et al. Markers of inflammation and cardiovascular disease: application to clinical and public health practice: A statement for healthcare professionals from the Centers for Disease Control and Prevention and the American Heart Association. Circulation. 2003;107(3):499-511.

5. Baigent C, Blackwell L, Emberson J, Holland LE, Reith C, Bhala $\mathrm{N}$, et al. Efficacy and safety of more intensive lowering of LDL cholesterol: a meta-analysis of data from 170,000 participants in 26 randomised trials. Lancet. 2010;376(9753):1670-81.

6. Ibanez B, James S, Agewall S, Antunes MJ, Bucciarelli-Ducci C, Bueno H, et al. 2017 ESC Guidelines for the management of acute myocardial infarction in patients presenting with STsegment elevation: The Task Force for the management of acute myocardial infarction in patients presenting with ST-segment elevation of the European Society of Cardiology (ESC). Eur Heart J. 2017;39(2):119-77.

7. Fang M, Qian Q, Zhao Z, Zhu L, Su J, Li X. High-Sensitivity CReactive Protein Combined with Low-Density Lipoprotein Cholesterol as the Targets of Statin Therapy in Patients with Acute Coronary Syndrome. Int Heart J. 2018;59(2):300-6.

8. Roffi M, Patrono C, Collet J-P, Mueller C, Valgimigli M, Andreotti F, et al. 2015 ESC Guidelines for the management of acute coronary syndromes in patients presenting without persistent ST-segment elevation: Task Force for the Management of Acute Coronary Syndromes in Patients Presenting without Persistent ST-Segment Elevation of the European Society of Cardiology (ESC). Eur Heart J. 2016;37(3):267-315.

9. Galkina E, Ley K. Immune and inflammatory mechanisms of atherosclerosis (*). Annu Rev Immunol. 2009;27:165-97.

10. Ma Y, Xiang C, Zhang B. Efficacy Evaluation of High-Dose Atorvastatin Pretreatment in Patients with Acute Coronary Syndrome: A Meta-Analysis of Randomized Controlled Trials. Med Sci Monit. 2018;24:9354-63.

11. O'Gara PT, Kushner FG, Ascheim DD, Casey DE Jr, Chung MK, de Lemos JA, et al. 2013 ACCF/AHA guideline for the management of ST-elevation myocardial infarction: a report of the American College of Cardiology Foundation/American Heart Association Task Force on Practice Guidelines. J Am Coll Cardiol. 2013;61(4):e78-140.

12. Anderson JL, Adams CD, Antman EM, Bridges CR, Califf RM, Casey DE Jr, et al. 2012 ACCF/AHA focused update incorporated into the ACCF/AHA 2007 guidelines for the management of patients with unstable angina/non-ST-elevation myocardial infarction: a report of the American College of Cardiology
Foundation/American Heart Association Task Force on Practice Guidelines. J Am Coll Cardiol. 2013;61(23):e179-347.

13. Catapano AL, Graham I, De Backer G, Wiklund O, Chapman MJ, Drexel H, et al. 2016 ESC/EAS Guidelines for the Management of Dyslipidaemias. Eur Heart J. 2016;37(39):2999-3058.

14. Grundy SM, Stone NJ, Bailey AL, Beam C, Birtcher KK, Blumenthal RS, et al. 2018 AHA/ACC/AACVPR/AAPA/ABC/ ACPM/ADA/AGS/APhA/ASPC/NLA/PCNA Guideline on the Management of Blood Cholesterol: A Report of the American College of Cardiology/American Heart Association Task Force on Clinical Practice Guidelines. Circulation. 2019;139(25): e1082-143.

15. Kumar B, Shah MAA, Kumar R, Kumar J, Memon A. Comparison of Atorvastatin and Rosuvastatin in Reduction of Inflammatory Biomarkers in Patients with Acute Coronary Syndrome. Cureus. 2019;11(6):e4898.

16. Pitt B, Loscalzo J, Monyak J, Miller E, Raichlen J. Comparison of lipid-modifying efficacy of rosuvastatin versus atorvastatin in patients with acute coronary syndrome (from the LUNAR study). Am J Cardiol. 2012;109(9):1239-46.

17. Faergeman O, Hill L, Windler E, Wiklund O, Asmar R, Duffield E, et al. Efficacy and tolerability of rosuvastatin and atorvastatin when force-titrated in patients with primary hypercholesterolemia: results from the ECLIPSE study. Cardiology. 2008;111(4):219-28.

18. Balci B. The modification of serum lipids after acute coronary syndrome and importance in clinical practice. Curr Cardiol Rev. 2011;7(4):272-6.

19. Pitt B, Loscalzo J, Ycas J, Raichlen JS. Lipid levels after acute coronary syndromes. J Am Coll Cardiol. 2008;51(15):1440-5.

20. Biasucci LM, Koenig W, Mair J, Mueller C, Plebani M, Lindahl $\mathrm{B}$, et al. How to use C-reactive protein in acute coronary care. Eur Heart J. 2013;34(48):3687-90.

21. Kubková L, Spinar J, Pávková Goldbergová M, Jarkovský J, Pařenica J. [Inflammatory response and C-reactive protein value in patient with acute coronary syndrome]. Vnitr Lek. 2013;59(11):981-8.

22. Khurana S, Gupta S, Bhalla H, Nandwani S, Gupta V. Comparison of anti-inflammatory effect of atorvastatin with rosuvastatin in patients of acute coronary syndrome. J Pharmacol Pharmacother. 2015;6(3):130-5.

23. Tunçez A, Altunkeser BB, Öztürk B, Ateş MS, Tezcan H, Aydoğan C, et al. Comparative effects of atorvastatin $80 \mathrm{mg}$ and rosuvastatin $40 \mathrm{mg}$ on the levels of serum endocan, chemerin, and galectin-3 in patients with acute myocardial infarction. Anatol J Cardiol. 2019;22(5):240-9.

24. Altunkeser BB, Tuncez A, Ozturk B, Tezcan H, Ates MS, Yilmaz C, et al. Comparative effects of high-dose atorvastatin versus rosuvastatin on lipid parameters, oxidized low-density lipoprotein, and proprotein convertase subtilisin kexin 9 in acute coronary syndrome. Coron Artery Dis. 2019;30(4):285-90.

25. Shah CP, Shah BP, Dani SI, Channa BB, Lakshmanan SS, Krishnamani NC, et al. Efficacy and safety of the intensive dose of rosuvastatin $40 \mathrm{mg} /$ day in patients with acute coronary syndrome and at high risk of cardiovascular diseaseROSUVEES-2. Indian Heart J. 2016;68(6):766-71.

26. Guo J, Zhang WZ, Zhao Q, Wo JS, Cai SL. Study on the effect of different doses of rosuvastatin on ventricular remodeling in patients with acute coronary syndrome after emergency percutaneous coronary intervention. Eur Rev Med Pharmacol Sci. 2017;21(19):4457-63. 